\title{
Functional magnetic resonance: biomarkers of response in breast cancer
}

\author{
Elizabeth AM O'Flynn* and Nandita M deSouza
}

\begin{abstract}
Functional magnetic resonance (MR) encompasses a spectrum of techniques that depict physiological and molecular processes before morphological changes are visible on conventional imaging. As understanding of the pathophysiological and biomolecular processes involved in breast malignancies evolves, newer functional MR techniques can be employed that define early predictive and surrogate biomarkers for monitoring response to chemotherapy. Neoadjuvant chemotherapy is increasingly used in women with primary breast malignancies to down-stage the tumour and enable successful breast conservation surgery. It also plays a role in the treatment of undetected micrometastases. Cardinal physiological features of tumours that occur as a result of interactions between cancer cells, stromal cells and secreted factors and cytokines and how they change with treatment provide the opportunity to detect changes in the tumour microenvironment prior to any morphological change. Through sequential imaging, tumour response can be assessed and non-responders can be identified early to enable alternative therapies to be considered. This review summarises the functional magnetic resonance biomarkers of response in patients with breast cancer that are currently available and under development. We describe the current state of each biomarker and explore their potential clinical uses and limitations in assessing treatment response. With the aid of selected interesting cases, biomarkers related to dynamic contrast-enhanced MRI, diffusion-weighted $\mathrm{MRI}, \mathrm{T} 2 * \mathrm{BOLD}$ and MR spectroscopy are described and illustrated. The potential of newer approaches, such as MR elastography, are also reviewed.
\end{abstract}

${ }^{*}$ Correspondence: Elizabeth.OFlynn@icr.ac.uk

Clinical Magnetic Resonance Group, Institute of Cancer Research, Royal Marsden NHS Foundation Trust, Sutton, Surrey, SM2 5PT, United Kingdom

\section{Introduction}

Magnetic resonance imaging (MRI) of the breast has an established role in assessing response to neoadjuvant chemotherapy and provides better monitoring of the chemotherapeutic effect than clinical breast examination, mammography and ultrasound, especially in non-mass lesions and tumours that have fragmented into many foci [1]. As the overall response rate offered by neoadjuvant chemotherapy ranges from $60 \%$ to $80 \%$, with complete pathological response rates being around $10 \%$ to $20 \%$ [2], identification of early response is important in planning subsequent management. More complex functional MRI techniques offer to quantify changes in tumour microvasculature, cell density, hypoxia, metabolism and stiffness and so provide early predictive and surrogate biological biomarkers for monitoring response to chemotherapy.

As breast tumours respond to chemotherapy, changes occur within the tumour and its microenvironment. Angiogenesis, the fundamental physiological process associated with tumour development, is interrupted. The composition of the extracellular matrix and stroma is altered, and secreted factors and cytokines, which can affect the transport of molecules to and from tumour cells, change the physiology and chemical composition of the tumour. For example, tumour cells become hypoxic and fragment, leaving fibrotic and collagenous tissue that may be quantified using functional magnetic resonance (MR) techniques.

This article reviews the functional MR biomarkers of response currently routinely available and under development for assessing treatment response. Specifically, dynamic contrast-enhanced (DCE)-MRI, diffusionweighted (DW)-MRI, intrinsic susceptibility-weighted MRI, MR spectroscopy (MRS) and MR elastography are described with a focus on the current state of each technique and its limitations as a response biomarker.

\section{Dynamic contrast enhanced MRI}

DCE-MRI of the breast involves an intravenous injection of a low molecular weight T1-shortening paramagnetic compound (a gadolinium chelate) at doses between 0.1 and $0.2 \mathrm{mmol} / \mathrm{kg}$. Agents currently licensed for use 
include gadopentetate dimeglumine (Magnevist), gadodiamide (Omniscan), gadobenate dimeglumine (Multihance), gadoteriodol (ProHance), gadofosveset trisodium (Vasovist), gadoxetic acid (Eovist) and gadoversetamide (OptiMARK). Once injected, gadolinium circulates in the blood stream before passing into the extravascular extracellular or interstitial space. The concentration of gadolinium equilibrates between the intravascular and extravascular compartments over time and is eventually excreted by the kidneys. Post contrast images provide additional information to the unenhanced sequences by exploiting differences in temporal enhancement characteristics between malignant and normal or benign tissues. The differential uptake and washout of gadolinium in each of these tissues results in an increased signal on T1-weighted $\left(\mathrm{T}_{1} \mathrm{~W}\right)$ images. Along with morphological assessment from the unenhanced $\mathrm{T}_{1} \mathrm{~W}$ and $\mathrm{T}_{2} \mathrm{~W}$ sequences, the use of gadolinium to depict enhancement characteristics of the tissues can improve the sensitivity of MRI for cancer detection to between $89 \%$ and $100 \%$ [3].

Along with its high sensitivity, the specificity of breast DCE-MRI, although initially reported to be low, has more recently been shown to equal that of mammography with significantly higher values than ultrasound [4]. The blooming sign seen in $63 \%$ of malignant compared to $14.7 \%$ of benign lesions describes a brisk enhancement with sharply shaped borders at 1 minute after a bolus contrast injection that become progressively unsharp [5]. Further potential adjunctive morphological indicators of malignancy include unifocal oedema (91\% of malignant lesions and $45 \%$ of benign lesions), centripetal enhancement with a rapidly enhancing outer ring that fills in (52\% malignant lesions [6]), and the hook sign, a hook-like connection to the underlying pectoral muscle $33 \%$ of malignant lesions and 5\% of benign lesions [7]). The presence of an adjacent vessel on subtraction images is also a promising sign for malignancy ( $85.9 \%$ of malignant and in situ lesions compared to $19.6 \%$ of benign lesions [8]). Finally, the addition of morphologic signs from unenhanced $\mathrm{T}_{2} \mathrm{~W}$ sequences, such as spiculated margins, homogeneous intermediate signal intensity or stellate appearance, have been described to further improve the specificity of breast MRI [8].

The rate of contrast uptake into breast lesions is nonlinear and differs between malignant and benign lesions (Figure 1) so that enhancement curve characteristics can be used in conjunction with morphologic features to aid differential diagnosis. Malignant lesions exhibit stronger and faster enhancement than benign changes or normal tissues. In benign lesions, a slow onset (type I) curve that plateaus after 3 to 5 minutes is described in $83 \%$ of cases. In malignant lesions, a rapid onset with plateau (type II) or rapid onset with washout (type III) curve can be found in $91 \%$ of cases (57\% for type III and $34 \%$ for type II) [9].

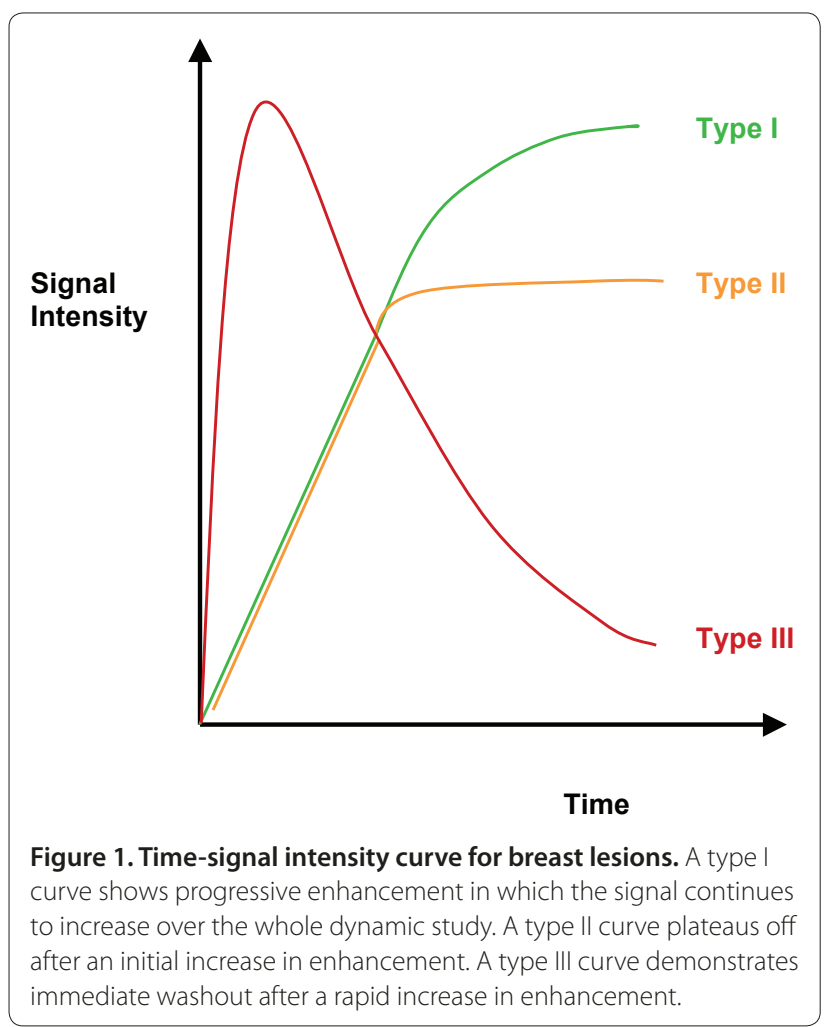

Semi-quantitative parameters can be calculated from these enhancement curves, including the onset time (from injection to the appearance of contrast in the tissues), maximum signal intensity, gradient or rate of contrast uptake and washout, and initial area under the time signal curve (IAUC).

Quantitative analysis involves pharmacokinetic modelling and requires more complex analysis methods of estimating changes in tissue contrast agent concentration following intravenous injection. Between $12 \%$ and $45 \%$ contrast leaks into the extravascular extracellular space $\left(\mathrm{v}_{\mathrm{e}}\right)$ during the first pass and results in measurable T1 shortening of tissues. The transfer constant, $\mathrm{K}^{\text {trans }}$, describes the transendothelial transport of contrast medium by diffusion from the vascular space to the tumour interstitium and provides a measure of vascular permeability. Over time, gadolinium diffuses back into the vasculature, which can be measured by the rate constant, $k_{\text {ep }}$. These parameters are related by the equation $\mathrm{k}_{\mathrm{ep}}=\mathrm{K}^{\mathrm{e}}$ trans $/ \mathrm{v}_{\mathrm{e}}$ [10] (Figure 2).

Magnetic field inhomogeneities induced by gadolinium on a $\mathrm{T}_{2} \mathrm{~W}$ image can also be exploited to derive relative measures of blood flow and volume (rBF and $\mathrm{rBV}$ ) as well as mean transit time (MTT). These variables are related by the central volume theorem equation $(\mathrm{BF}=\mathrm{BV} / \mathrm{MTT})$. The signal loss on a $\mathrm{T}_{2} \mathrm{~W}$ sequence caused by dephasing of spins is related to the concentration of gadolinium and thus to vessel size and density [11]. 


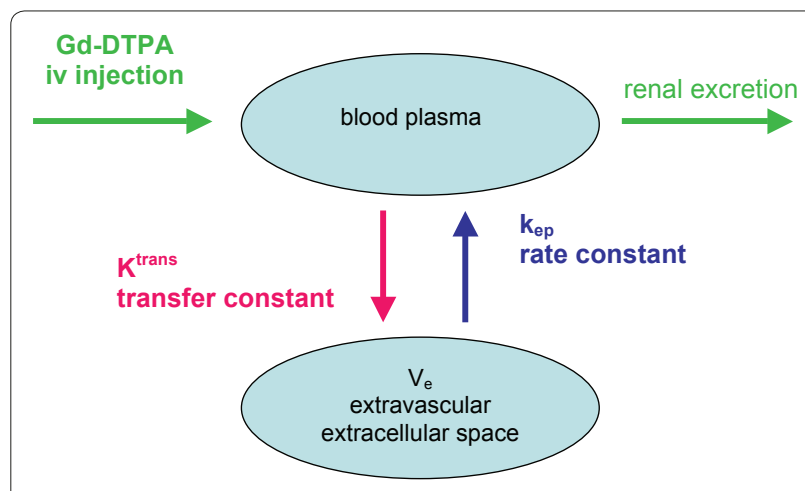

Figure 2. Distribution of gadolinium diethylenetriamine pentaacetic acid (Gd-DTPA) in body compartments after intravenous (iv) injection.

There is good evidence for use of such quantitative measures in breast cancer. $K^{\text {trans }}$ is generally high in tumours, as is $\mathrm{k}_{\mathrm{ep}}$ (Fig. 3), and a significant reduction of up to a third has been shown in both parameters in patients with locally advanced breast cancer responding early in their course of neoadjuvant chemotherapy [12]. In addition, an increase in $\mathrm{v}_{\mathrm{e}}$ of nearly a third has been shown in non-responders [13]. rBV and rBF obtained from $\mathrm{T}_{2}{ }^{*} \mathrm{~W}$ dynamic susceptibility sequences have also shown reductions of around two-thirds in patients responding to treatment [12]; however, $\mathrm{T}_{2}{ }^{*} \mathrm{~W}$ functional imaging, while commonly used in MRI of the brain, is almost never used in breast MRI. Alterations in these parameters are likely to relate to changes in microvessel density and function of the microvasculature due to antiangiogenic effects of chemotherapy. The evidence from phase I and II studies strongly suggests that $\mathrm{K}^{\text {trans }}$ can be used as a predictive biomarker to determine response to antiangiogenic drugs or vascular disruptive agents, with a change in $\mathrm{K}^{\text {trans }}$ of greater than $40 \%$ considered by many investigators as the threshold required to represent definitive disease response [14]. There is some discrepancy in the published data, however, with several other studies demonstrating little or no decrease in $K^{\text {trans }}$ or $k_{\text {ep }}$ following neoadjuvant chemotherapy [15,16]; in fact, one small study of 29 patients scanned very early after one cycle of chemotherapy showed that early tumour size change is a better response predictor than either $\mathrm{K}^{\text {trans }}$ or $\mathrm{k}_{\mathrm{ep}}$ [17].

The explanation for these variations in reported data are multifactorial: patient numbers, tumour type, chemotherapeutic agent and time-point of scanning after commencing therapy have all varied. The classification of responders was also not consistent, varying from a $65 \%$ reduction in the largest tumour volume [13] to the accepted Response Evaluation Criteria in Solid Tumours (RECIST) or International Union Against Cancer criteria [18] of $30 \%$ or more reduction in one-dimensional tumour size or a $50 \%$ or more reduction in the product of the tumour diameter (assuming a spherical tumour model), respectively $[12,15,16]$. The group who demonstrated change in tumour size to be a better predictor of response compared to $\mathrm{K}^{\text {trans }}$ or $\mathrm{k}_{\mathrm{ep}}$ chose an arbitrary reduction of $15 \%$ in one-dimensional size [17].

There was also a significant difference in data analysis methodology; most studies used manual regions-ofinterest (ROI) on enhanced subtracted images, although one group used semi-automated ROI generation, and in addition analysed a $3 \times 3$ pixel ROI hot spot [13]. Furthermore, the median or mean of each pharmacokinetic parameter for analysis has not been consistent. An increasing awareness of the heterogeneity of breast tumours makes the median a more appropriate parameter, with the change in the skewness of the distribution of these parameters likely to be as significant as changes in the median value.

Another source of variation is the range of mathematical models used for pharmacokinetic analysis and the choice of arterial input function measurement, which also impacts on the overall results of tumour vascular heterogeneity. Traditionally, use of a nearby blood vessel for arterial contrast was deemed the ideal arterial input function [19], but population-based arterial input functions are more robust [20]. Alternatively, tumour enhancement relative to that in neighbouring muscle tissue can be evaluated [21], and avoids error from flow effects in blood vessels. With the introduction of standardised scanning protocols, automated analysis software and the publication of reproducibility studies, derivation of pharmacokinetic parameters could become more standardised and robust and be usefully adopted as functional imaging markers in breast cancer.

\section{Diffusion-weighted MRI}

DW-MRI develops intrinsic contrast within tissues based on the microscopic motion of water molecules by applying magnetic field gradients during the MRI pulse sequence that sensitize the readout signal to losses from this motion. DW-MRI contrast provides different, and complementary, information to DCE-MRI, being sensitive to factors that affect this microscopic water motion, such as cell density, membrane integrity and tissue microstructure. Changes in signal intensity on DW-MRI reflect the movement of water diffusion over distances of 0 to $30 \mu \mathrm{m}$ over time periods of 50 to $100 \mathrm{~ms}$ [22]. As with other tumours, breast cancers demonstrate restricted diffusion because water molecules cannot move as freely in tissues with a high cell density where extracellular space is limited (Figure 4); this results in reduced signal loss from Brownian motion and is seen as a high signal intensity lesion on the DW-MRI image (Figure 5). 
(a)(b)(c)

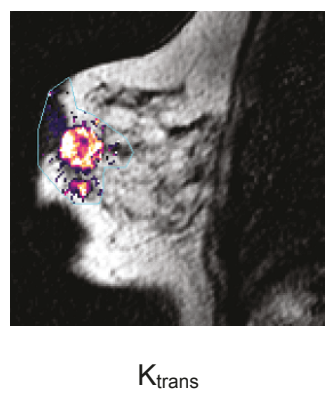

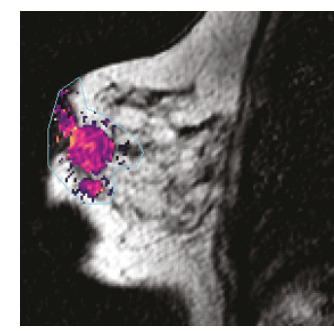

$\mathrm{v}_{\mathrm{e}}$

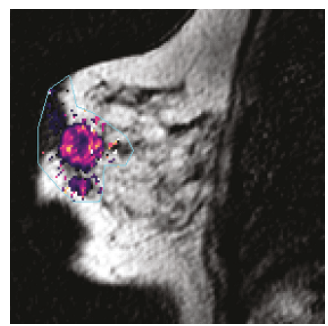

$k_{\mathrm{ep}}$

Figure 3. Images showing pharmacokinetic modelling parameters. (a-c) Malignant tumour within the breast illustrated on dynamic contrastenhanced MRI and using the vascular parameters: (a) transfer constant ( $\left.K^{\text {trans }}\right)$; (b) extravascular extracellular space $\left(\mathrm{V}_{\mathrm{e}}\right)$; (c) rate constant from extravascular/extracellular space back into plasma $\left(\mathrm{k}_{\mathrm{ep}}\right)$. All these parameters are higher at the tumour periphery compared to the centre and in the satellite nodule, indicative of more neoangiogenesis in these areas.

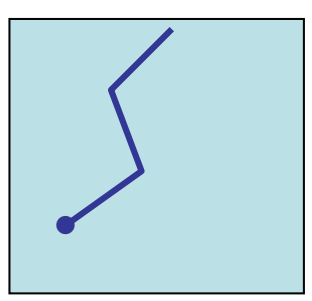

Random

Brownian Motion

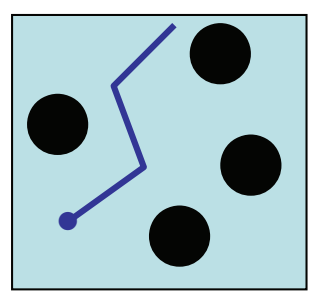

Free diffusion Low signal intensity DWI High ADC

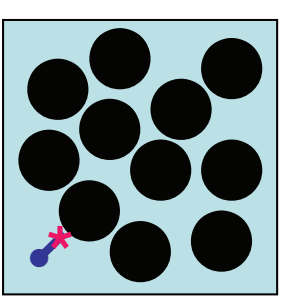

Restricted diffusion

High signal intensity DWI Low ADC

- water molecule

cell

Figure 4. Diagram illustrating free and restricted diffusion of water in different tissues. ADC, apparent diffusion coefficient; DWI, diffusion weighted imaging.

Magnetic field gradients used to provide diffusion weighting can be varied in their amplitude, duration and spacing, which are jointly reflected by a 'b' value. Acquisition of DW-MRI data using at least two b values allows calculation of an apparent diffusion coefficient (ADC), a derived logarithmic parameter of signal change with $b$ value. At very low b values $\left(<100 \mathrm{~s} / \mathrm{mm}^{2}\right)$, the ADC predominantly reflects larger distances of water movement likely to represent movement within microvessels. This phenomenon is known as intravoxel incoherent motion [23]. By eliminating these low b values, this 'perfusion' effect in vascular rich areas can be suppressed and the ADC value can more accurately represent the shorter distances travelled by water protons in the extracellular space, or true diffusion [24].

The role of DW-MRI in tumour diagnosis is gradually being explored and it is increasingly shown to aid decision-making [25]. Differentiation between malignant and benign breast lesions using DW-MRI has been well reported [26-28], with the mean ADC value of malignant lesions being significantly lower than that of benign lesions or normal breast tissue. This degree of overlap requires incorporation of an ADC threshold methodology for analysis; a $1.6 \times 10^{-3} \mathrm{~mm}^{2} / \mathrm{s}$ cutoff gives up to $96 \%$ sensitivity and $55 \%$ specificity for tumour identification [27]. More recently, one group has normalised ADC values to the surrounding glandular tissue and demonstrated a reduction in overlap between benign and malignant lesions. Using this method, normalised ADC values for tumour and benign lesions are $0.55 \times 10^{-3} \mathrm{~mm}^{2} / \mathrm{s}$ and $1.1 \times 10^{-3} \mathrm{~mm}^{2} / \mathrm{s}$, respectively) [29], with the optimal threshold of $1.6 \times 10^{-3} \mathrm{~mm}^{2} / \mathrm{s}$ normalising to $0.7 \times 10^{-3} \mathrm{~mm}^{2} / \mathrm{s}$.

The visibility of lesions on DW-MRI is better in older women compared to younger women, likely related to the density of the glandular parenchyma. Also, due to the lower spatial resolution offered by DW-MRI compared to DCE-MRI, the diagnostic performance of DW-MRI is less helpful for non-mass-like lesions such as invasive lobular carcinomas and lesions $<1 \mathrm{~cm}$ in size [30]. The 
(a)(b)

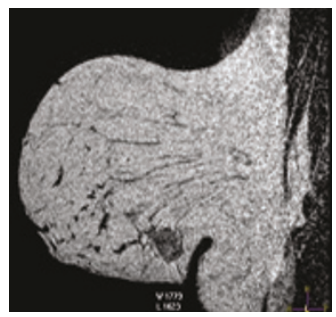

$\mathrm{T}_{2} \mathrm{~W}$ image

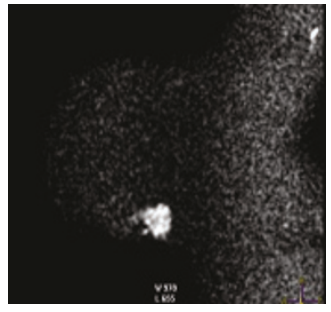

DWI image $b=200$

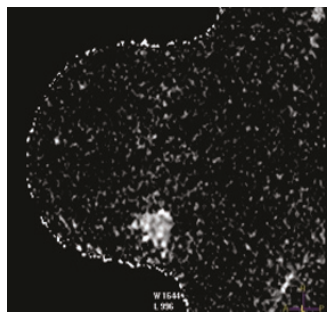

ADC map

Figure 5. Diffusion weighted images of a breast tumour. (a) Sagittal $T_{2} W$ image through the breast shows a well-defined lobulated mass inferiorly. (b) This appears as a bright area of restricted diffusion on the corresponding diffusion weighted MRI (DW-MRI) image (b = 200). (c) The calculated apparent diffusion coefficient (ADC) map shows the heterogeneity of diffusion coefficient values within the tumour.

major false-positive lesions reported are intraductal papillomas and fibrocystic disease, which can also result in overestimation of cancer extension [27]. Mucinous carcinomas interestingly demonstrate a significantly higher ADC compared to other types of breast cancer, leading to false-negative reports [31].

DW-MRI also shows promise as an early surrogate biomarker for detecting response to neoadjuvant chemotherapy. Induction of successful apoptosis results in loss of membrane integrity, alteration of membrane barriers to water diffusion and cell shrinkage, increasing extracellular space. This translates to a rise in the ADC value of up to $35 \%$ and precedes any decrease in tumour size in locally advanced tumours [32-34]. Transient early decreases in ADC have also been demonstrated before this increase, and are thought to be related to cell swelling, reduction in blood flow or changes in composition of the extracellular space [16].

The optimal b values for diffusion-weighted MRI in the breast have not been established; nor indeed have the optimal scanning protocol, imaging parameters and methods of analysis, which all have a bearing on the ADC value. Published data indicate that b values from 0,600 to 850 , and up to 1,000 may be optimal [27,28,30], with at least three values required to ensure robustness of reproducibility of the measurement. The ability to obtain these data without the use of extrinsic contrast agents, in a short scanning time, independent of magnetic field strength and operator interpretation is hugely advantageous. Reproducibility studies and quality assurance of methodology crucially need to be established.

\section{$\mathrm{T}_{2}{ }^{*}$ /blood oxygen level-dependent MRI}

Blood oxygen level-dependent (BOLD) or intrinsic susceptibility-weighted MRI relies on the paramagnetic property of deoxyhaemoglobin, which creates susceptibility variations in the magnetic field (or microscopic field gradients), which in turn decrease the transverse relaxation rate $R_{2}^{*}\left(=1 / T_{2}^{*}\right)$ of water in blood and the tissue surrounding blood vessels. An increase in the deoxyhaemoglobin concentration (that is, hypoxia) leads to a decrease in the signal intensity on the $\mathrm{T}_{2}{ }^{*}$ image and a faster $R_{2}^{*}$ [35] (Figure 6). An improvement in oxygenation has the converse effect. Deoxyhaemoglobin therefore acts as an intrinsic BOLD contrast agent for imaging tissue hypoxia. Specific gradient-recalled echo (GRE) sequences are required to detect changes in $\mathrm{R}_{2}{ }^{*}$. Variations in $\mathrm{R}_{2}^{*}$ have largely been evaluated in xenograft and human models using inhaled carbogen $\left(95 \%\right.$ oxygen $\left(\mathrm{O}_{2}\right)$, $5 \%$ carbon dioxide $\left.\left(\mathrm{CO}_{2}\right)\right)$ to intensify the otherwise small changes in signal intensity: the $\mathrm{CO}_{2}$ induces vasodilation and the $\mathrm{O}_{2}$ tension is high with $95 \% \mathrm{O}_{2}$ so that subtracted images with and without carbogen reveal regions of hypoxia where signal change is greatest. Unfortunately, the hyperventilation induced by breathing carbogen in humans is poorly tolerated so reliance has been largely on $\mathrm{R}_{2}{ }^{*}$ measurements during air, or alternatively $100 \%$ oxygen, breathing.

A recent study in breast cancer patients has shown $\mathrm{R}_{2}^{*}$ values to be significantly lower in tumour than normal breast parenchyma prior to the commencement of chemotherapy [36], suggesting that breast tumours are less hypoxic than normal breast tissue, possibly because of their high vascularity. This contrasts with other recently published data in prostate cancer [37], where $R_{2}^{*}$ is increased, indicating increased hypoxia in these tumours. The increased $\mathrm{R}_{2}^{*}$ in normal breast tissue has also been related to the fibrocollagenous ligaments of Cooper, which maintain normal breast structural integrity and contribute to higher $\mathrm{R}_{2}{ }^{*}$ values. In responders following treatment, the $\mathrm{R}_{2}{ }^{*}$ value has been shown to increase, likely as a result of decreased blood flow; however, in this one published study this parameter was not as efficacious as changes in other DCE-MRI parameters, such as $\mathrm{K}^{\text {trans, }}$ $\mathrm{rBV}$, and $\mathrm{rBF}$, or even morphological parameters such as tumour size, in indicating response [36]. The complexity 
(a)

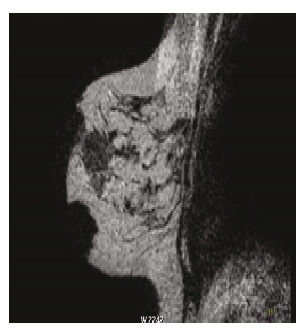

T2W image (b) (c)

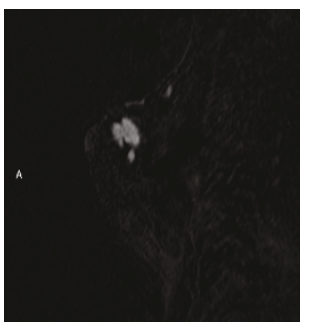

DCE Subtraction image

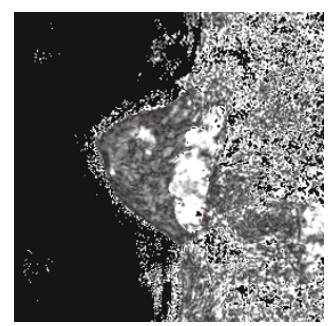

$\mathrm{T}_{2}{ }^{*} \mathrm{~W}$ image

Figure 6. $T_{2}{ }^{*} W$ images of a breast tumour. (a-c) Sagittal T2W (a), dynamic contrast-enhanced (DCE) subtracted image at time point 2.44 minutes (b), and $\mathrm{T}_{2}^{*} \mathrm{~W}$ image (c) in a patient with a palpable breast lump in the upper outer quadrant. The tumour seen in (a) and highlighted in (b) shows heterogeneity of $\mathrm{T}_{2}^{*}$ with faster signal decay in the inferior part of the tumour, indicating a greater deoxyhaemoglobin content here.

and heterogeneity of the microvasculature in different tumour types thus need to be understood prior to using such measurements for evaluating changes in tumour oxygenation in response to chemotherapy.

\section{MR spectroscopy}

MRS exploits the nuclear spin properties of hydrogen $\left({ }^{1} \mathrm{H}\right)$ as well as of other atoms with unpaired protons, such as ${ }^{31} \mathrm{P},{ }^{23} \mathrm{Na}$ and ${ }^{19} \mathrm{~F}$, in a magnetic field to absorb and emit radiofrequency. The acquired frequency spectrum of a range of metabolites provides information about the altered metabolism of cancer cells. With ${ }^{1} \mathrm{H}$ MRS, efficient water suppression is mandatory to document proton resonances within molecules known to be increased in cancer, such as choline and lipids; protons within these molecules resonate at slightly different frequencies when placed in a magnetic field because of their immediate molecular environment. In breast cancer, as with other tumours, high levels of cholinecontaining metabolites involved in phospholipid metabolism, and thus cellular membranes in proliferation, result in a triplet at $3.22 \mathrm{ppm}$ of free choline, phosphocholine and glycerophosphocholine. Choline is virtually undetectable in normal breast tissue and a peak at $3.25 \mathrm{ppm}$ indicates benign tissue [38].

Several groups have shown that total choline concentration [Cho] can be used as a marker of malignancy, and when combined with DCE-MRI, increases the specificity of breast MR up to $88 \%$ (and to $100 \%$ after the inclusion of a single slice $\mathrm{T}_{2}{ }^{*}$ perfusion measurement) [39]. In vivo ${ }^{1} \mathrm{H}-\mathrm{MRS}$ has also been shown to be useful in monitoring metabolic response to chemotherapy, with increased [Cho] and water/fat ratios in malignant tumours indicative of residual disease [40]. Small patient studies to date show promising results, with reduction of the choline signal following two cycles of neoadjuvant chemotherapy being more sensitive than changes in tumour size at predicting pathological response [41]. Also, by imaging after one cycle of chemotherapy, the same group demonstrated that a reduction in the choline signal may be more sensitive than DW-MRI in demonstrating pathological response [41,42].

In vivo ${ }^{1} \mathrm{H}$ MRS is a single, large voxel technique, and overall variations in the fat and water composition, particularly in heterogenous tumours such as invasive lobular cancers and ductal carcinoma in situ, reduces sensitivity of [Cho] quantification. Partial volume effects in a large voxel also cause problems in the quantification of [Cho], which pose a significant problem after neoadjuvant chemotherapy [43].

Two dimensional localised correlated sepctroscopy (L-COSY MRS) incorporates a second spectral dimension that is indirectly detected through the acquisition of multiple one-dimensional MRS with incrementally longer times to echo (TEs). Cross-correlation of peaks enables identification of lipid species by reducing contamination from overlapping metabolite resonances. Early reports have shown identification of invasive ductal carcinoma within these spectra versus normal fatty breast tissue with $92.4 \%$ sensitivity and $92.7 \%$ specificity [44]. However, the technique is time consuming (20 minutes for a $3 \mathrm{~cm}$ voxel) and requires specialist analysis software, so its clinical utility is limited.

Sodium $\left({ }^{23} \mathrm{Na}\right)$ MRI has also been shown to be a very sensitive indicator of cellular integrity and cellular energy metabolism [45], with an elevated tissue sodium concentration in neoplastic tissue. ${ }^{23} \mathrm{Na}$ images can be accurately determined by co-registering high-resolution ${ }^{1} \mathrm{H}$ images acquired during the same scan. Its potential as a surrogate biomarker of response has been reported with a significantly reduced tissue sodium concentration in responders after one cycle of chemotherapy [46].

\section{MR elastography}

MR elastography, an imaging correlate to palpation, is another novel technique that can be easily implemented 
in the clinic. It assesses the viscoelastic shear properties of lesions by direct MRI visualisation of acoustic waves and quantifies the decreased elasticity of malignant tumours. Quantification of the differential 'stiffness' between a breast lesion and the background adipose and fibroglandular tissues is achieved by assessing the propagation of mechanical waves, generated by an electromechanical driver, through the breast using a gradient echo phase contrast sequence [47]. The tissue stiffness map (or elastogram) is based on a linear scale, calibrated into kilopascals and represented as a colour map.

MR elastography can be performed as an extension to conventional breast MRI and could potentially be incorporated into a standard MRI breast examination. It is already being used clinically for the assessment of patients with chronic liver disease. There have been very few studies of breast MR elastography; early published data on a small population group suggests that MR elastography in combination with DCE-MRI could increase the diagnostic performance of breast MRI and increase its specificity from $75 \%$ with a persistently high sensitivity of $90 \%$ [48]. Further investigations of larger cohorts and smaller lesions will be necessary to validate these results.

\section{Discussion}

In patients with breast cancer, traditional anatomical imaging using size and morphological criteria for assessing response to neoadjuvant chemotherapy does not provide information on functional changes within tumours. The current challenge is to move beyond these anatomical boundaries to develop a more personalised, individual assessment of functional changes within a tumour for response evaluation. For those with the poorest prognosis and most advanced disease, early identification of nonresponders allows alternative management options to be considered. MRI has the versatility to contribute to the functional assessment of breast tumours and improve diagnostic confidence, as well as provide early surrogate markers of disease response.

Functional MRI biomarkers of response described need careful validation, ideally against clinical outcome measures, before they can be adopted as established surrogate end points of response. However, currently an insufficient number of clinical studies have been reported with this kind of data for this to happen; reported changes are summarised in Table 1. Multicentre validation against histopathological markers, such as for microvessel density, apoptosis, hypoxia and vascularity, would further qualify the use of these functional biomarkers and support their translation into clinical practice. However, histological validation lacks true 'functional' input, so the limitation of this kind of validation needs to be recognised: it may well be that MRI on its own may more accurately reflect in vivo tumour physiology.

In an attempt to tackle the issues of validation, two phase II multicentre national trials are underway - NeoCOMICE in the United Kingdom and I-SPY 2 in the US, both of which are examining the effectiveness of MRI in the early prediction of response to neoadjuvant chemotherapy and the development of surrogate imaging biomarkers. Neo-COMICE, currently in its pilot stage, also aims to evaluate the optimal scanning protocols that determine parameters of greatest predictive value of treatment response and establish parity of MRI examiations between centres. Currently, there is no consensus on a standardized MR imaging examination or on the role of MRI for assessing response in patients receiving neoadjuvant chemotherapy. The adaptive design of the I-SPY 2 trial will allow early data from one set of patients to guide decisions about which treatments may be more useful in the trial in order to eliminate ineffective treatments and offer patients the best chance of successful therapy [49].

The increased availability of higher-field MRI scanners allows higher signal-to-noise ratio and so better spatial resolution to increase the visibility of small cancers. Early reports suggest that the sensitivity and specificity of DCE-MRI at 3T for malignant breast lesions increase to $95 \%$ and $91 \%$, respectively, from $91 \%$ and $85 \%$ [50]. Going up in field strength from the commonly available $1.5 \mathrm{~T}$ to $3.0 \mathrm{~T}$ is not without challenges, however, as the nonuniformity of the magnetic induction field $\left(B_{1}\right)$ results in non-homogeneity of fat suppression, which in turn leads to poor enhancement in areas with a very low magnetic induction field and errors in quantifying enhancement ratios. Reports have shown that the $\mathrm{B}_{1}$ field in one breast can be reduced by as much as $40 \%$, which is sufficient to reduce the conspicuity of a malignant lesion and reduce the sensitivity to cancer detection [51]. Quantitative functional MRI at 3T requires improved radiofrequency excitation methods and improved analysis to ensure $B_{1}$ inhomogeneity is accounted for when calculating DCE metrics.

Future potential in the search for biomarkers of efficacy for certain therapeutic treatments may lie in correlating baseline gene expression with MRI response using several of the above techniques. Gene arrays and immunohistochemistry analysis of vascular endothelial growth factor pathways could indicate which pattern of gene expression relates to specific changes in vascular volume and permeability assessed by MRI, and this is a promising area of research [52].

Ongoing research and recent technical advances indicate that the prospects for substantial improvements in monitoring of therapeutic response as well as for improved early detection and accurate diagnosis of breast 
Table 1. Reported changes quantified on functional magnetic resonance following neoadjuvant chemotherapy for breast cancer

\begin{tabular}{|c|c|c|c|}
\hline Functional technique and study & Responders & Non-responders & $\begin{array}{c}\text { Time point imaged } \\
\text { after NAC }\end{array}$ \\
\hline \multicolumn{4}{|c|}{ DCE-MRI (percentage change in parameters) } \\
\hline \multirow[t]{4}{*}{ Ah-See et al. [12] } & $K^{\text {trans }} \downarrow 39.8 \%$ & $K^{\text {trans }} \uparrow 18.1 \%$ & 2 cycles \\
\hline & $k_{\text {ep }} \downarrow 33.3 \%$ & $\mathrm{k}_{\mathrm{ep}} \uparrow 7.4 \%$ & 2 cycles \\
\hline & $\mathrm{rBV} \downarrow 59.3 \%$ & rBV $\uparrow 73.4 \%$ & 2 cycles \\
\hline & $\mathrm{rBF} \downarrow 56.4 \%(n=19)$ & $\mathrm{rBF} \uparrow 70.6 \%(n=19)$ & 2 cycles \\
\hline \multirow[t]{3}{*}{ Pickles et al. [13] } & $K^{\text {trans }} \downarrow 19.7 \%$ & $K^{\text {trans }} \downarrow 19.6 \%$ & 'At early time point' \\
\hline & $k_{\text {ep }} \downarrow 19.9 \%$ & $k_{e p} \downarrow 36.2 \%$ & \\
\hline & $v_{e} \uparrow 4.36 \%(n=48)$ & $v_{e} \uparrow 27.6 \%(n=20)$ & \\
\hline \multirow[t]{2}{*}{ Padhani et al. [15] } & $K^{\text {trans }} \downarrow 22 \%(n=9)$ & $K^{\text {trans }} \downarrow 6.5 \%(n=6)$ & 1 cycle \\
\hline & $K^{\text {trans }} \downarrow 62 \%(n=7)$ & $K^{\text {trans }} \downarrow 25 \%(n=6)$ & 2 cycles \\
\hline
\end{tabular}

DW-MRI (percentage change in ADC)

Pickles et al. [32]

Sharma et al. [33]

Nilsen et al. [34]

$\mathrm{R}_{2}^{*}$ (change in $\mathrm{s}^{-1}$ )

Li et al. [36]

MRS (percentage change in Cho SNR)

Jacobs et al. [46]

$$
\begin{aligned}
& \uparrow 16 \%(n=10) \\
& \uparrow 27 \%(n=10) \\
& \uparrow 15 \%(n=14) \\
& \uparrow 27 \%(n=24) \\
& \uparrow 35 \%(n=29) \\
& \uparrow 25 \%(n=25)
\end{aligned}
$$

$\uparrow 10 \%(n=27)$
1 cycle

2 cycles

1 cycle

2 cycles

3 cycles

4 cycles

${ }^{23} \mathrm{Na} \mathrm{MRI}$ (percentage change in total sodium concentration)

Jacobs et al. [46]

$\downarrow 27 \%(n=15)$

$\downarrow 21 \%(n=3)$

1 cycle

ADC, apparent diffusion coefficient; Cho, choline; DCE, dynamic contrast-enhanced; DW, diffusion-weighted; MRI, magnetic resonance imaging; MRS, magnetic resonance spectroscopy; NA, sodium; NAC, neoadjuvant chemotherapy; rBF, relative blood flow; rBV, relative blood volume; SNR, signal-to-noise ratio.

cancer with functional MRI are promising, with the rate of development indicating early translation to routine clinical care. A key factor in their success will depend on rigorous quality control and assurance to ensure that the quantitative measurements are robust and reproducible.

\section{Abbreviations}

ADC, apparent diffusion coefficient; BOLD, blood oxygen level dependent; DCE, dynamic contrast-enhanced; DW, diffusion-weighted; MR, magnetic resonance; $\mathrm{MRI}$, magnetic resonance imaging; $\mathrm{MRS}$, magnetic resonance spectroscopy; rBF, relative blood flow; rBV, relative blood volume; $\mathrm{ROI}$, region of interest.

\section{Competing interests}

The authors declare that they have no competing interests.

Published: 23 February 2011

\section{References}

1. Sardanelli F, Boetes C, Borisch B, Decker T, Federico M, Gilbert FJ, Helbich T, Heywang-Köbrunner SH, Kaiser WA, Kerin MJ, Mansel RE, Marotti L, Martincich L, Mauriac L, Meijers-Heijboer H, Orecchia R, Panizza P, Ponti A, Purushotham $A D$, Regitnig P, Del Turco MR, Thibault F, Wilson R: Magnetic resonance imaging of the breast: recommendations from the EUSOMA working group. Eur J Cancer 2010, 46:1296-1316.

2. Swain SM, Sorace RA, Bagley CS, Danforth DN Jr, Bader J, Wesley MN, Steinberg SM, Lippman ME: Neoadjuvant chemotherapy in the combined modality approach of locally advanced nonmetastatic breast cancer. Cancer Res 1987, 47:3889-3894.

3. Kuhl CK: Current status of breast MR imaging. Part 2. Clinical applications. Radiology 2007, 244:672-691.

4. Warner E, Plewes DB, Shumak RS, Catzavelos GC, Di Prospero LS, Yaffe MJ, Goel V, Ramsay E, Chart PL, Cole DE, Taylor GA, Cutrara M, Samuels TH, Murphy JP, Murphy JM, Narod SA: Comparison of breast magnetic resonance imaging, mammography, and ultrasound for surveillance of women at high risk for hereditary breast cancer. J Clin Oncol 2001, 
19:3524-3531

5. Fischer DR, Baltzer P, Malich A, Wurdinger S, Freesmeyer MG, Marx C, Kaiser WA: Is the "blooming sign" a promising additional tool to determine malignancy in MR mammography? Eur Radio/ 2004, 14:394-401.

6. Jinguji M, Kajiya Y, Kamimura K, Nakajo M, Sagara Y, Takahama T, Ando M, Rai Y, Sagara Y, Ohi Y, Yoshida H: Rim enhancement of breast cancers on contrast-enhanced MR imaging: relationship with prognostic factors. Breast Cancer 2006, 13:64-73.

7. Malich A, Fischer DR, Wurdinger S, Boettcher J, Marx C, Facius M, Kaiser WA: Potential MRI interpretation model: differentiation of benign from malignant breast masses. AJR Am J Roentgenol 2005, 185:964-970.

8. Fischer DR, Malich A, Wurdinger S, Boettcher J, Dietzel M, Kaiser WA: The adjacent vessel on dynamic contrast-enhanced breast MRI. AJRAm J Roentgenol 2006, 187:W147-W151

9. Kuhl CK, Mielcareck P, Klaschik S, Leutner C, Wardelmann E, Gieseke J, Schild $\mathrm{HH}$ : Dynamic breast MR imaging: are signal intensity time course data useful for differential diagnosis of enhancing lesions? Radiology 1999, 211:101-110.

10. Tofts PS, Brix G, Buckley DL, Evelhoch JL, Henderson E, Knopp MV, Larsson HB, Lee TY, Mayr NA, Parker GJ, Port RE, Taylor J, Weisskoff RM: Estimating kinetic parameters from dynamic contrast-enhanced T(1)-weighted MRI of a diffusable tracer: standardized quantities and symbols. J Magn Reson Imaging 1999, 10:223-232.

11. Padhani AR: Dynamic contrast-enhanced MRI in clinical oncology: current status and future directions. J Magn Reson Imaging 2002, 16:407-422.

12. Ah-See ML, Makris A, Taylor NJ, Harrison M, Richman PI, Burcombe RJ, Stirling JJ, d'Arcy JA, Collins DJ, Pittam MR, Ravichandran D, Padhani AR: Early changes in functional dynamic magnetic resonance imaging predict for pathologic response to neoadjuvant chemotherapy in primary breast cancer. Clin Cancer Res 2008, 14:6580-6589.

13. Pickles MD, Lowry M, Manton DJ, Gibbs P, Turnbull LW: Role of dynamic contrast enhanced MRI in monitoring early response of locally advanced breast cancer to neoadjuvant chemotherapy. Breast Cancer Res Treat 2005, 91:1-10.

14. O'Connor JP, Jackson A, Parker GJ, Jayson GC: DCE-MRI biomarkers in the clinical evaluation of antiangiogenic and vascular disrupting agents. $\mathrm{Br} J$ Cancer 2007, 96:189-195.

15. Padhani AR, Hayes C, Assersohn L, Powles T, Makris A, Suckling J, Leach MO, Husband JE: Prediction of clinicopathologic response of breast cancer to primary chemotherapy at contrast-enhanced MR imaging: initial clinical results. Radiology 2006, 239:361-374.

16. Manton DJ, Chaturvedi A, Hubbard A, Lind MJ, Lowry M, Maraveyas A, Pickles MD, Tozer DJ, Turnbull LW: Neoadjuvant chemotherapy in breast cancer: early response prediction with quantitative MR imaging and spectroscopy. Br J Cancer 2006, 94:427-435.

17. Yu HJ, Chen JH, Mehta RS, Nalcioglu O, Su MY: MRI measurements of tumor size and pharmacokinetic parameters as early predictors of response in breast cancer patients undergoing neoadjuvant anthracycline chemotherapy. J Magn Reson Imaging 2007, 26:615-623.

18. Hayward JL, Rubens RD, Carbone PP, Heuson JC, Kumaoka S, Segaloff A: Assessment of response to therapy in advanced breast cancer. A project of the programme on clinical oncology of the International Union against Cancer, Geneva, Switzerland. Eur J Cancer 1978, 14:1291-1292.

19. Henderson E, Rutt BK, Lee TY: Temporal sampling requirements for the tracer kinetics modeling of breast disease. Magn Reson Imaging 1998 16:1057-1073

20. Parker GJ, Roberts C, Macdonald A, Buonaccorsi GA, Cheung S, Buckley DL, Jackson A, Watson Y, Davies K, Jayson GC: Experimentally-derived functional form for a population-averaged high-temporal-resolution arterial input function for dynamic contrast-enhanced MRI. Magn Reson Med 2006, 56:993-1000.

21. Kovar DA, Lewis M, Karczmar GS: A new method for imaging perfusion and contrast extraction fraction: input functions derived from reference tissues. J Magn Reson Imaging 1998, 8:1126-1134.

22. Norris DG: The effects of microscopic tissue parameters on the diffusion weighted magnetic resonance imaging experiment. NMR Biomed 2001, 14:77-93.

23. Le BD, Breton E, Lallemand D, Aubin ML, Vignaud J, Laval-Jeantet M: Separation of diffusion and perfusion in intravoxel incoherent motion MR imaging. Radiology 1988, 168:497-505

24. Padhani AR, Liu G, Koh DM, Chenevert TL, Thoeny HC, Takahara T, Dzik-Jurasz
A, Ross BD, Van Cauteren M, Collins D, Hammoud DA, Rustin GJ, Taouli B, Choyke PL: Diffusion-weighted magnetic resonance imaging as a cancer biomarker: consensus and recommendations. Neoplasia 2009, 11:102-125.

25. deSouza NM, Riches SF, Vanas NJ, Morgan VA, Ashley SA, Fisher C, Payne GS, Parker C: Diffusion-weighted magnetic resonance imaging: a potential non-invasive marker of tumour aggressiveness in localized prostate cancer. Clin Radio/ 2008, 63:774-782.

26. Woodhams R, Matsunaga K, Kan S, Hata H, Ozaki M, Iwabuchi K, Kuranami M Watanabe $M$, Hayakawa K: ADC mapping of benign and malignant breast tumors. Magn Reson Med Sci 2005, 4:35-42.

27. Partridge SC, DeMartini WB, Kurland BF, Eby PR, White SW, Lehman CD: Differential diagnosis of mammographically and clinically occult breast lesions on diffusion-weighted MRI. J Magn Reson Imaging 2010, 31:562-570

28. Bogner W, Gruber S, Pinker K, Grabner G, Stadlbauer A, Weber M, Moser E, Helbich TH, Trattnig S: Diffusion-weighted MR for differentiation of breast lesions at 3.0 T: how does selection of diffusion protocols affect diagnosis? Radiology 2009, 253:341-351.

29. Ei Khouli RH, Jacobs MA, Mezban SD, Huang P, Kamel IR, Macura KJ, Bluemke DA: Diffusion-weighted imaging improves the diagnostic accuracy of conventional 3.0-T breast MR imaging. Radiology 2010, 256:64-73.

30. Partridge SC, DeMartini WB, Kurland BF, Eby PR, White SW, Lehman CD: Quantitative diffusion-weighted imaging as an adjunct to conventional breast MRI for improved positive predictive value. AJR Am J Roentgeno 2009, 193:1716-1722.

31. Hatakenaka M, Soeda H, Yabuuchi H, Matsuo Y, Kamitani T, Oda Y, Tsuneyoshi M, Honda H: Apparent diffusion coefficients of breast tumors: clinical application. Magn Reson Med Sci 2008, 7:23-29.

32. Pickles MD, Gibbs P, Lowry M, Turnbull LW: Diffusion changes precede size reduction in neoadjuvant treatment of breast cancer. Magn Reson Imaging 2006, 24:843-847

33. Sharma U, Danishad KK, Seenu V, Jagannathan NR: Longitudinal study of the assessment by MRI and diffusion-weighted imaging of tumor response in patients with locally advanced breast cancer undergoing neoadjuvant chemotherapy. NMR Biomed 2009, 22:104-113.

34. Nilsen L, Fangberget A, Geier O, Olsen DR, Seierstad T: Diffusion-weighted magnetic resonance imaging for pretreatment prediction and monitoring of treatment response of patients with locally advanced breast cancer undergoing neoadjuvant chemotherapy. Acta Oncol 2010, 49:354-360.

35. Robinson SP, Howe FA, Rodrigues LM, Stubbs M, Griffiths JR: Magnetic resonance imaging techniques for monitoring changes in tumor oxygenation and blood flow. Semin Radiat Oncol 1998, 8:197-207.

36. Li SP, Taylor NJ, Makris A, Ah-See ML, Beresford MJ, Stirling JJ, d'Arcy JA, Collins DJ, Padhani AR: Primary human breast adenocarcinoma: imaging and histologic correlates of intrinsic susceptibility-weighted MR imaging before and during chemotherapy. Radiology 2010, 257:643-652.

37. Alonzi R, Padhani AR, Maxwell RJ, Taylor NJ, Stirling JJ, Wilson Jl, d'Arcy JA, Collins DJ, Saunders MI, Hoskin PJ: Carbogen breathing increases prostate cancer oxygenation: a translational MRI study in murine xenografts and humans. Br J Cancer 2009, 100:644-648.

38. Tozaki M: Proton MR spectroscopy of the breast. Breast Cancer 2008, 15:218-223.

39. Huang W, Fisher PR, Dulaimy K, Tudorica LA, O'Hea B, Button TM: Detection of breast malignancy: diagnostic MR protocol for improved specificity. Radiology 2004, 232:585-591.

40. Jagannathan NR, Kumar M, Seenu V, Coshic O, Dwivedi SN, Julka PK Srivastava A, Rath GK: Evaluation of total choline from in-vivo volume localized proton MR spectroscopy and its response to neoadjuvant chemotherapy in locally advanced breast cancer. Br J Cancer 2001, 84:1016-1022

41. Tozaki M, Sakamoto M, Oyama Y, Maruyama K, Fukuma E: Predicting pathological response to neoadjuvant chemotherapy in breast cancer with quantitative $1 \mathrm{H}$ MR spectroscopy using the external standard method. J Magn Reson Imaging 2010, 31:895-902.

42. Tozaki M, Oyama Y, Fukuma E: Preliminary study of early response to neoadjuvant chemotherapy after the first cycle in breast cancer: comparison of $1 \mathrm{H}$ magnetic resonance spectroscopy with diffusion magnetic resonance imaging. Jpn J Radiol 2010, 28:101-109.

43. Su MY, Baik HM, Yu HJ, Chen JH, Mehta RS, Nalcioglu O: Comparison of choline and pharmacokinetic parameters in breast cancer measured by MR spectroscopic imaging and dynamic contrast enhanced MRI. Technol Cancer Res Treat 2006, 5:401-410. 
44. Thomas MA, Wyckoff N, Yue K, Binesh N, Banakar S, Chung HK, Sayre J, DeBruhl N: Two-dimensional MR spectroscopic characterization of breast cancer in vivo. Technol Cancer Res Treat 2005, 4:99-106.

45. Ouwerkerk R, Jacobs MA, Macura KJ, Wolff AC, Stearns V, Mezban SD, Khouri NF, Bluemke DA, Bottomley PA: Elevated tissue sodium concentration in malignant breast lesions detected with non-invasive $23 \mathrm{Na}$ MRI. Breast Cancer Res Treat 2007, 106:151-160.

46. Jacobs MA, Stearns V, Wolff AC, Macura K, Argani P, Khouri N, Tsangaris T, Barker PB, Davidson NE, Bhujwalla ZM, Bluemke DA, Ouwerkerk R: Multiparametric magnetic resonance imaging, spectroscopy and multinuclear $((2)(3) \mathrm{Na})$ imaging monitoring of preoperative chemotherapy for locally advanced breast cancer. Acad Radio/ 2010 17:1477-1485

47. McKnight AL, Kugel JL, Rossman PJ, Manduca A, Hartmann LC, Ehman RL: MR elastography of breast cancer: preliminary results. AJR Am J Roentgenol 2002, 178:1411-1417.

48. Siegmann KC, Xydeas T, Sinkus R, Kraemer B, Vogel U, Claussen CD: Diagnostic value of MR elastography in addition to contrast-enhanced MR imaging of the breast-initial clinical results. Eur Radiol 2010, 20:318-325.

49. Barker AD, Sigman CC, Kelloff GJ, Hylton NM, Berry DA, Esserman LJ: I-SPY 2: an adaptive breast cancer trial design in the setting of neoadjuvant chemotherapy. Clin Pharmacol Ther 2009, 86:97-100

50. Lo GG, Ai V, Chan JK, Li KW, Cheung PS, Wong TT, Ma M, Lee R, Chien D: Diffusion-weighted magnetic resonance imaging of breast lesions: first experiences at 3 T. J Comput Assist Tomogr 2009, 33:63-69.

51. Azlan CA, Di GP, Ahearn TS, Semple SI, Gilbert FJ, Redpath TW: B1 transmission-field inhomogeneity and enhancement ratio errors in dynamic contrast-enhanced MRI (DCE-MRI) of the breast at 3T. J Magn Reson Imaging 2010, 31:234-239.

52. Mehta S, Li SP, Hughes NP, Adams RF, Padhani AR, Buffa FM, Wright CA, McRae $L A$, Makris A, Harris AL: Investigation of pathways regulating early antiangiogenic response to bevacizumab given prior to neoadjuvant breast cancer chemotherapy. J Clin Oncol 2010, 28 (suppl; abstr TPS176):15s.

doi: $10.1186 /$ bcr 2815

Cite this article as: O'Flynn EAM, deSouza NM: Functional magnetic resonance: biomarkers of response in breast cancer. Breast Cancer Research 2011, 13:204 